\title{
Calcium Oscillations Index the Extent of Calcium Loading and Predict Functional Recovery during Reperfusion in Rat Myocardium
}

\author{
Robert G. Weiss, Gary Gerstenblith, and Edward G. Lakatta \\ Laboratory of Cardiovascular Science, Gerontology Research Center, National Institute on Aging, Baltimore, Maryland 21224; and \\ Cardiology Division, Department of Medicine, Johns Hopkins Hospital, Baltimore, Maryland 21205
}

\begin{abstract}
Delayed recovery of contractile function after myocardial ischemia may be due to prolonged recovery of high-energy phosphates, persistent acidosis, increased inorganic phosphate, and/or calcium loading. To examine these potential mechanisms, metabolic parameters measured by ${ }^{31} \mathrm{P}$ nuclear magnetic resonance spectroscopy, and spontaneous diastolic myofilament motion caused by sarcoplasmic reticulum-myofilament calcium cycling indexed by the scattered light intensity fluctuations (SLIF) it produces in laser beam reflected from the heart, were studied in isolated atrioventricularly blocked rat hearts $(n=10)$ after $65 \mathrm{~min}$ of ischemia at $30^{\circ} \mathrm{C}$. All metabolic parameters recovered to their full extent $5 \mathrm{~min}$ after reperfusion. Developed pressure evidenced a small recovery but then fell abruptly. This was accompanied by an increase in end diastolic pressure to $37 \pm 5 \mathrm{~mm} \mathrm{Hg}$ and a fourfold increase in SLIF, to $252 \pm 58 \%$ of baseline. In another series of hearts initial reperfusion with calcium of $0.08 \mathrm{mM}$ prevented the SLIF rise and resulted in improved developed pressure (74 $\pm 3 \%$ vs. $39 \pm 13 \%$ of control), and lower cell calcium $(5.9 \pm 3$ vs. 10.3 $\pm 1.4 \mu \mathrm{mol} / \mathrm{g}$ dry wt). Thus, during reperfusion, delayed contractile recovery is not associated with delayed recovery of pH, inorganic phosphate, or high-energy phosphates and can be attributed, in part, to an adverse effect of calcium loading which can be indexed by increased SLIF occurring at that time. (J. Clin. Invest. 1990. 85:757-765.) diastolic calcium oscillations $\bullet$ reperfusion $\bullet$ high-energy phosphates
\end{abstract}

\section{Introduction}

Previously ischemic myocardium often exhibits delayed mechanical recovery and as such is referred to as "stunned" myocardium $(1,2)$. Although the duration of ischemia is one factor that influences the degree of stunning, there are several additional potential contributors including delayed recovery of high-energy phosphates consumed during ischemia, delayed correction of acidosis, negative effects of inorganic phosphate, and/or deleterious calcium overload occurring during reperfusion (3-12). To study the significance of these factors during early the postischemic recovery period, phosphorus-31 nuclear magnetic resonance (NMR) ${ }^{1}$ spectroscopy was used to nonin-

Address reprint requests to Dr. Lakatta, Laboratory of Cardiovascular Science, Gerontology Research Center, National Institute on Aging, 4940 Eastern Avenue, Baltimore, MD 21224.

Received for publication 25 January 1989 and in revised form 3 October 1989

1. Abbreviations used in this paper: EDP, end diastolic pressure; NMR, nuclear magnetic resonance; $\mathrm{PCr}$, phosphocreatine; $\mathrm{P}_{\mathrm{i}}$, inorganic phosphate; SLIF, scattered light intensity fluctuations.

The Journal of Clinical Investigation, Inc.

Volume 85, March 1990, 757-765 vasively and repetitively quantify high-energy phosphates (ATP and phosphocreatine [PCr]), inorganic phosphate $\left(\mathbf{P}_{i}\right)$, and intracellular $\mathrm{pH}(13)$ and spectroscopy of scattered laser light was used to study spontaneous diastolic sarcoplasmic reticulum-myofilament calcium oscillations $(14,15)$. Spontaneous, asynchronous, myocardial calcium oscillations, which do not require membrane depolarization, have been shown to occur in various cardiac preparations in mammalian species $(16,17)$. These have been attributed to calcium-dependent calcium release from the sarcoplasmic reticulum and exhibit a periodicity which depends on cell calcium loading (18). The inhomogeneous contractile motion caused by the spontaneous oscillations phase modulates a laser beam passed through or backscattered from the cardiac tissue, producing intensity fluctuations in the scattered light (SLIF), which are measured in hertz. In the setting of normal perfusion, excessive oscillations occur during conditions of cellular calcium loading and are associated with altered diastolic and systolic function (19-22).

To evaluate the contributions of these potential causes of delayed contractile recovery after a myocardial ischemic insult, we have studied the isolated beating rat heart model using these techniques in the settings of ischemia/reperfusion and exposure to altered perfusate calcium during normal perfusion and during the postischemic reperfusion period.

\section{Methods}

Heart preparation. Retired, male breeder Wistar rats, weighing 500-650 g, received heparin, $1000 \mathrm{U}$, and were anesthetized with $50-100 \mathrm{mg}$ of sodium pentobarbital, both by intraperitoneal injection. After rapid excision of the heart, each aorta was cannulated for retrograde perfusion at $30^{\circ} \mathrm{C}$ with a constant flow of $15 \mathrm{ml} / \mathrm{min}$ delivered by a peristaltic pump (Masterflex 7562-10, Cole-Parmer Instrument Co. Barrington, IL) (Fig. $1 A$ ). A flow integrator (VRW Scientific Div., San Francisco, CA) was placed in the line to eliminate pressure oscillations produced by the peristaltic pump. Hearts were perfused with oxygenated solution $(\mathrm{pH}=7.4)$ containing, in millimolar: sodium, 144; potassium, 5; calcium, 1.5; Hepes, 6; magnesium, 0.9; chloride, 152; glucose, 15 ; and lidocaine, $5 \mu \mathrm{g} / \mathrm{ml}$ added for control of ventricular arrhythmias. Hearts that were later assayed for ionic contents were perfused with $1 \mathrm{mM} \mathrm{K}^{+} \mathrm{Co}^{+} \mathrm{EDTA}$, which was used as an extracellular marker, and the $\mathrm{KCl}$ was reduced to $4 \mathrm{mM}$. In some protocols, perfusate calcium was lowered; this was not accompanied by adjustments in other cations. Hearts which were studied at slowed heart rates also received propranolol $1 \mu \mathrm{g} / \mathrm{ml}$ and atrioventricular heart block. Successful atrioventricular conduction block, obtained by surgical incision into the interatrial septum via the atrial appendages, was readily assessed by visual inspection with heart rate slowing from $\sim 180$ beats/ $\min$ to $<20$ beats/min. Each atrioventricularly blocked heart was paced at a rate of 20 beats/min with a stimulator (model SD-9, Grass Instrument Co., Quincy, $\mathrm{MA}$ ) via a $\mathrm{KCl}$ wick electrode placed into the right ventricle and secured to the pulmonary artery outflow. A latex balloon attached to PE 190 tubing (Becton, Dickinson \& Co., Parsippany, NJ) was inserted into the left ventricle through the mitral valve and filled with aqueous solution to obtain an initial end diastolic pressure (EDP) of $10-16 \mathrm{~mm} \mathrm{Hg}$. Throughout the experiment the 

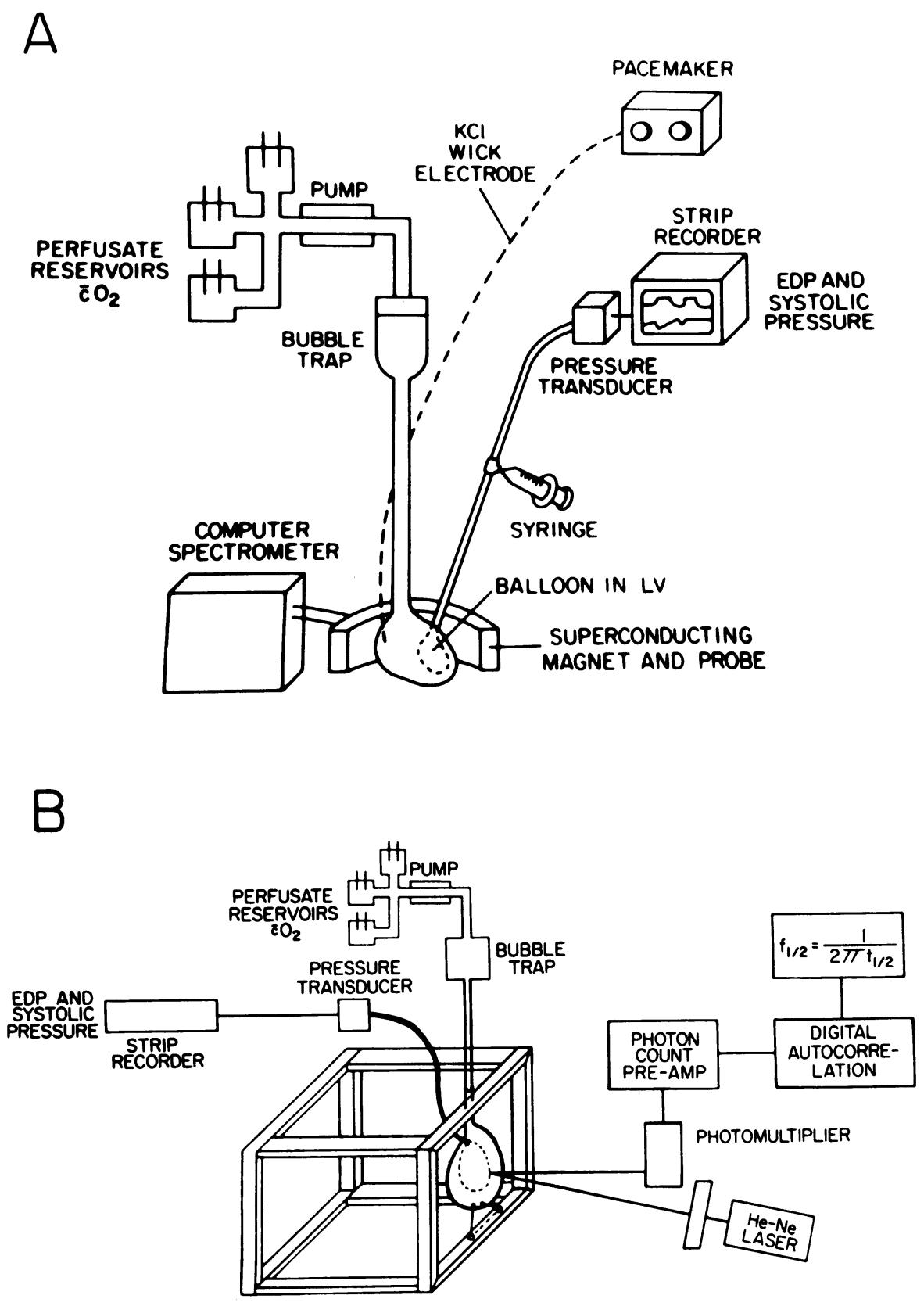

Figure 1. Sketches of the isolated rat heart models. $(A)$ Representation of a heart within the bore of the 4.2-T NMR magnet. Constant-flow perfusion is maintained by the roller pump with oxygenated solution. Isovolumic pressure is continuously measured by the intraventricular balloon connected to a strip recorder. $(B)$ Schematic diagram of a heart studied with the laser backscattering apparatus. The laser light is incident upon the surface of the left ventricle and the light backscattered at $30^{\circ}$ is collected by a pinhole and directed to the surface of the photomultiplier tube. Isovolumic pressure is measured as in the NMR experiments. See Methods for additional details.

balloon volume was not altered except when noted and thus the heart functioned isovolumically. The other end of the PE 190 tubing was connected to a transducer (Statham model no. P23D6, Gould Medical Products, Oxnard, CA) wired to a direct writing recorder (Gould Brush) for continuous measurements of left ventricular pressure.

${ }^{31} P$ NMR Spectroscopy. The hearts studied with NMR spectroscopy were positioned in a $25-\mathrm{mm}$ probe of a superconducting magnet (field strength 4.2 T, model WH-180, Bruker Instruments, Inc., Billerica, MA) interfaced with a computer (model 1280, Nicolet Instrument Corp., Madison, WI) (Fig. $1 \mathrm{~A}$ ). Pulsed, Fourier-transformed spectra were obtained with a 2-s delay between pulses of $28-\mu$ s duration $\left(65^{\circ}\right.$ flip angle). Data were accumulated during 2.5- or 5-min acquisitions with a $4 \mathrm{~K}$ table and $3,000-\mathrm{Hz}$ spectral width. Proton decoupling was not used. Changes in tissue contents of $P_{i}, P C r$, and ATP were determined by integrating areas under individual peaks from summed 5- or 10-min acquisitions for each heart with a digitizer (model 9810, Hewlett-Packard Co., Palo Alto, CA). Previous studies indicate that changes in $P_{i}, P C r$, and ATP assessed by these NMR techniques corre- late well with direct biochemical quantification (23-25). The aqueous solution within the latex balloon contained trimetaphosphate as a standard. Intracellular $\mathrm{pH}$ was measured from the chemical shift of the $P_{i}$ peak relative to the $\mathrm{PCr}$ peak in 5-min spectra (26).

Laser backscatter experiments. The method for quantitating SLIF has been previously described by Stern et al. $(14,15)$. Briefly, hearts studied with laser backscattering were positioned in a water-jacketed glass container at $30^{\circ} \mathrm{C}$ with the left ventricular surface adjacent to the anterior window (Fig. $1 B$ ). Light from a $5 \mathrm{~mW} \mathrm{He}-\mathrm{Ne}$ laser $(\lambda=632.8$ $\mathrm{nm}$ ), which had been polarized by a combination of filters and collimated by a 1-mm pinhole, was incident on the left ventricular surface through this window. Light backscattered from the heart at an angle of $30^{\circ}$ was collimated by a $1-\mathrm{mm}$ pinhole, passed through another horizontal polarizing lens, and collected through another pinhole $(600 \mu \mathrm{m})$ at the surface of a photomultiplier tube (FW130, ITT Electo Optical Products, Fort Wayne, IN). This arrangement was designed to produce an effective object which was circular and constant in size. The output was analyzed by a digital autocorrelator (model K7025, Malverne In- 
struments, Southborough, MA) for which averaging times, prescale levels and time "gating" sequences were automatically determined by a computer (9825A, Hewlett-Packard Co.) to obtain a precision of 5\% (27). SLIF measurements are presented as $f_{1 / 2} ; f_{1 / 2}=1 /(2 \pi)\left(t_{1 / 2}\right)$ where $t_{1 / 2}$ is the time for the autocorrelation function to decay by $50 \%$. In the present study, a modified time gating protocol was used to avoid systolic motion artifact and consisted of recording SLIF measurements during the last second of diastole in hearts continuously stimulated 20 times per minute. The total collection time for each SLIF measurement was 1-1.5 min as required to determine a valid autocorrelation function and as determined by the computer program (19).

Cell ionic quantification. $\mathrm{K}^{+} \mathrm{Co}^{+} \mathrm{EDTA}, 1 \mathrm{mM}$ was used as an extracellular marker (28-30) in certain experiments and synthesized in pure crystalline form by the method of Dwyer et al. (31). Immediately after the experiments, wet weights of pieces of left ventricular free wall were obtained and dry weights after at least $48 \mathrm{~h}$ at $110^{\circ} \mathrm{C}$. All tissues were handled with and stored in teflon instruments and containers. The dried tissue was digested with nitric and perchloric acids and the total tissue calcium, cobalt, and sodium were ascertained by flame atomic absorption spectrophotometry. The extracellular space $(E C S)$ was calculated by the following formula: $E C S=\mathrm{Co}_{\mathrm{t}} /\left(\mathrm{Co}_{\mathrm{p}}\right)\left(\mathrm{H}_{2} \mathrm{O}\right)$, where $\mathrm{Co}_{t}$ is the total tissue cobalt, $\mathrm{Co}_{\mathrm{p}}$ is the perfusate cobalt concentration, and $\mathrm{H}_{2} \mathrm{O}$ is total tissue water in milliliters obtained from the dry weight assuming $1 \mathrm{ml}$ of $\mathrm{H}_{2} \mathrm{O}=1 \mathrm{~g}$.

The intracellular ionic concentration was calculated by subtracting the extracellular ionic content from the total content: $C_{\mathrm{i}}=$ total - extracellular; $C_{\mathrm{i}}=C_{\mathrm{t}}-(E C S)\left(\mathrm{H}_{2} \mathrm{O}\right)\left(C_{\mathrm{p}}\right)$, where $C_{\mathrm{i}}$ is the cellular ion content, $C_{\mathrm{t}}$ is the total tissue ion content, and $C_{\mathrm{p}}$ is the perfusate concentration of the ion. The intracellular ionic contents are expressed as micromole/gram dry weight.

Protocol. Initially, all hearts underwent a stabilization period while perfused with oxygenated solution. Subsequently, baseline data were collected for $20 \mathrm{~min}$. To ensure uniformity, hearts were excluded from the study protocol if developed pressure was $<100 \mathrm{~mm} \mathrm{Hg}$. To assess stability of the experimental preparation three hearts were studied for at least $80 \mathrm{~min}$ after the baseline period following atrioventricular block at a heart rate of 20 beats/min.

To assess the time course of recovery of mechanical function and metabolism after a 40-min, global no-flow, ischemic insult at a "physiologic" heart rate of 200 beats/min in the absence of propranolol and surgical atrioventricular block, a group of five hearts was studied using NMR spectroscopy under these experimental conditions.

\section{Results}

Effect of ischemia and reflow on contractile and metabolic function during pacing at 200 beats/min in the absence of atrioventricular block and propranolol. A typical tracing of a heart studied at pacing rates of 200 beats/min is shown in Fig. 2. Developed pressure rapidly falls during ischemia. After reper- fusion there is an early rise in developed pressure but then a subsequent fall before a more gradual recovery to its final value. The mean \pm SEM $(n=5)$ preischemic developed pressure of $170 \pm 4$ was rapidly abolished, and EDP increased from $12 \pm 2$ to $28.2 \pm 3 \mathrm{~mm} \mathrm{Hg}$ at the end of the 40 -min ischemic period. Mean developed pressure during reperfusion, as percentage of baseline values, is shown in Fig. $3 \mathrm{~A}$. During the first 3 min developed pressure climbs rapidly but recovery is then slowed and developed pressure actually falls at 3-4 min of reperfusion from $65 \pm 9 \%$ at $3 \mathrm{~min}$ to $56 \pm 7 \%$ at $5 \mathrm{~min}$. The fall in developed pressure after initial recovery occurred in four of five hearts during minutes 3-6 of reperfusion and no heart demonstrated a rise in developed pressure during minutes 3-4. Recovery of developed pressure subsequently continues at a slower rate than previously and eventually attains $97 \%$ of baseline developed pressure by $25 \mathrm{~min}$. EDP rises to $33 \pm 8 \mathrm{~mm} \mathrm{Hg}$ during early reperfusion peaking between minutes 2 and 4 and falls slowly thereafter to $14 \pm 2 \mathrm{~mm} \mathrm{Hg}$ at $25 \mathrm{~min}$.

Simultaneous NMR data were collected during the above experiments at the stimulation rate of 200 beats/min. Typical spectra are shown in Fig. 4. The mean preischemic $\mathrm{PCr} / \mathrm{P}_{\mathrm{i}}$ ratio was $9.1 \pm 1.4$, corrected for partial saturation effects, in these hearts. During ischemia a rapid fall in $\mathrm{PCr}$ and a slow decline in ATP are noted. $P_{i}$ rises rapidly and the chemical shift moves downfield as intracellular acidosis develops. Fig. 3 $B$ shows the average integrated NMR data as percentage of baseline preischemic values vs. reperfusion time where time $=0$ represents the data obtained at the end of ischemia. A rapid reversal in $\mathrm{pH}(5.88 \pm 0.14$ to $7.17 \pm 0.03)$ is noted and $\mathrm{pH}$ returns to normal within $5 \mathrm{~min}$. PCr and ATP also plateau at their eventual levels by $5 \mathrm{~min}$, attaining $115 \pm 4 \%$ and $68 \pm 4 \%$ of their respective control values. Therefore, delayed mechanical recovery during the first 3-5 min of reperfusion may in part be attributed to delayed recovery of $\mathrm{P}_{\mathrm{i}}, \mathrm{pH}, \mathrm{PCr}$, and ATP. However, the decline in percent developed pressure at 5 min and the continued slow eventual recovery, which is not completed until $25 \mathrm{~min}$ of reperfusion, cannot be explained by delayed recovery of these global metabolic parameters because levels of these have recovered to their fullest eventual extent by 5-7 min of reperfusion.

SLIF measurements during ischemia and reflow. The use of SLIF measurements to examine the contribution of altered calcium loading to delayed contractile recovery could not be studied at the high stimulation rates. The contractile and NMR measurements were therefore repeated in another series of five hearts studied at a lower heart rate obtained by ventric-

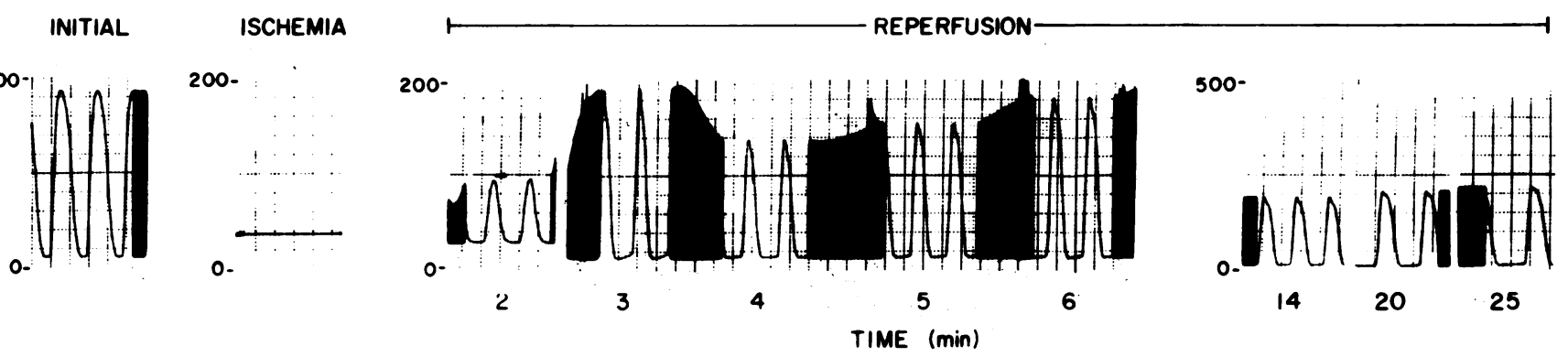

Figure 2. Contractile recovery during reperfusion at a heart rate of 200 beats/min. A representative recording of intraventricular isovolumic pressure during baseline, $\mathbf{4 0}$ min of global, no-flow ischemia, and sequentially during reperfusion. Note the early rise in developed pressure during the first 3 min of reperfusion but the subsequent transient fall in developed pressure (minutes $3-4)$ before a slower increase to the final recovery value. 


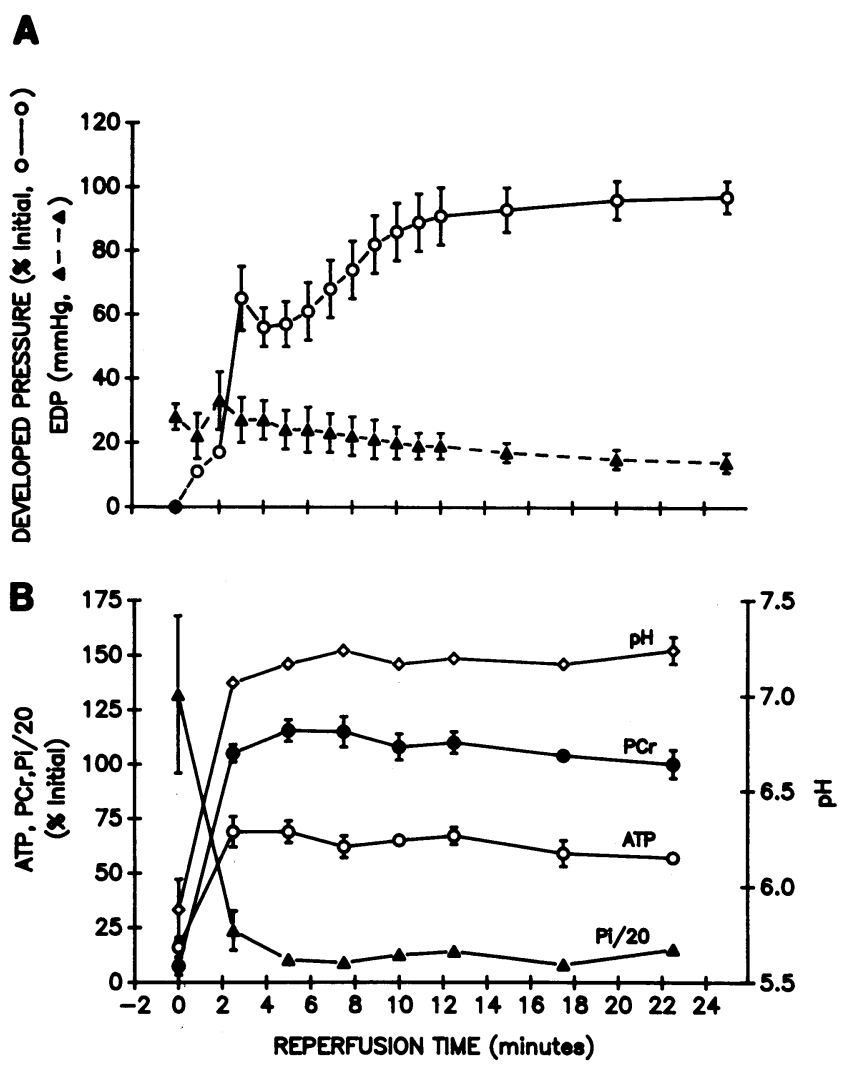

Figure 3. Contractile and metabolic recovery during reperfusion at a heart rate of 200 beats/min. (A) Average developed pressure as percentage of baseline and EDP $(\mathrm{mm} \mathrm{Hg})$. $(B)$ Mean values of PCr, ATP, and $P_{i}$, as percentage of preischemic values, and intracellular $\mathrm{pH}$. Spectra were not summed from separate hearts. Each point represents the mean \pm SEM for five hearts. Metabolic parameters recover to their eventual levels within $5 \mathrm{~min}$ but contractile recovery does not recover to its eventual level until 15-20 min.

BASELINE

5 MIN REPERFUSION

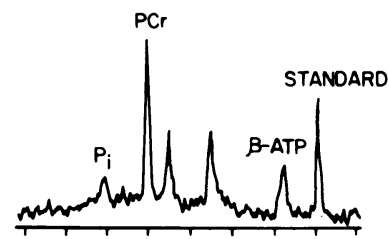

END-ISCHEMIA
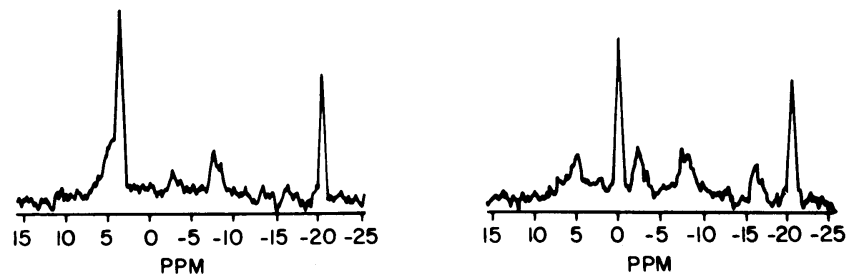

Figure 4. Phosphorus-31 NMR spectra from a representative heart at a heart rate of $200 / \mathrm{min}$. Typical ${ }^{31} \mathrm{P}$ NMR spectra obtained during baseline, at the end of $40 \mathrm{~min}$ of ischemia, and during early $(5 \mathrm{~min})$ and late $(30 \mathrm{~min})$ reperfusion demonstrating the peak areas of $\mathrm{PCr}$, $\beta$-ATP, and $\mathrm{P}_{\mathrm{i}}$. Initial mean $\mathrm{PCr} / \mathrm{ATP}$ and $\mathrm{P}_{\mathrm{i}} / \mathrm{ATP}$ ratios, after correction for partial saturation, were $2.11 \pm 0.14$ and $0.24 \pm 0.07$, respectively. Note that levels of high energy phosphates and $\mathrm{pH}$ have recovered to their full extent as early as 5 minutes after reperfusion. ular pacing in the presence of surgical atrioventricular block and propranolol. The ischemic duration was lengthened to 65 min to approximate, as assessed by mechanical function and high-energy phosphates, the previous ischemic insult since the lower heart rate and presence of propranolol diminished the metabolic and functional changes induced during a 40-min ischemic period. Hearts were excluded from study if significant tachyarrhythmias, which prevented pacing at a rate of 20 beats/min, occurred during the $40-$ min reperfusion period. Parallel experiments were then conducted under identical experimental conditions in another group of five hearts using the laser apparatus (Fig. $1 B$ ). In these, simultaneous SLIF, developed pressure, and EDP were recorded during the course of 65 min of global, no-flow, ischemia and $40 \mathrm{~min}$ of reperfusion. A representative example is shown in Fig. 5. Since the functional results were similar for the spectroscopy and laser experiments the mechanical data are combined for all 10 hearts studied.

Mean developed pressure, which was initially $173 \pm 7 \mathrm{~mm}$ $\mathrm{Hg}$, rapidly decreased during ischemia, as was true at the higher heart rate in the previous experiments, and a late rise in EDP from $12 \pm 2$ to $24 \pm 2$ occurred. Fig. $6 A$ shows the developed pressure recovery as percent baseline during reperfusion for all 10 of these hearts studied at a heart rate of 20 beats $/ \mathrm{min}$. Developed pressure rises and peaks at $5 \mathrm{~min}$ at $32 \pm 4 \%$ of baseline but then falls to $18 \pm 5 \%$ at $7.5 \mathrm{~min}$. Subsequently, developed pressure rises slowly and plateaus at $100 \pm 5 \mathrm{~mm} \mathrm{Hg}$, $59 \pm 4 \%$ of its initial value. The fall in developed pressure after 5 min of reflow occurred in 8 of 10 hearts and only two hearts evidenced a consistently rising developed pressure throughout reperfusion. EDP rises initially and peaks by $7.5 \mathrm{~min}$ at $36 \pm 3$ $\mathrm{mm} \mathrm{Hg}$ and falls slowly thereafter, plateauing at $27 \pm 4 \mathrm{~mm} \mathrm{Hg}$. Initial SLIF was $19.3 \pm 2.2 \mathrm{~Hz}$ and declined to 0 during ischemia. These results during pacing at 20 beats/min are similar to those reported in the arrested heart model (32). SLIF recovery as percent of baseline during reperfusion is superimposed on the simultaneous developed pressure and EDP tracings in Fig. $6 \mathrm{~A}$. During early reperfusion SLIF exhibit a rapid rise at 5 min and peak in all hearts between 7 and 8 min at $252 \pm 58 \%$ of baseline. Thereafter, mean SLIF frequency gradually decreases and is only $57 \pm 9 \%$ of the initial value at $40 \mathrm{~min}$.

The results of the simultaneous metabolic data for the five NMR-studied hearts are presented in Fig. $6 C$ and are similar to the results presented in Fig. 3 obtained at the higher heart rate. The mean preischemic $\mathrm{PCr} / \mathrm{P}_{\mathrm{i}}$ ratio for these hearts was 21.7 \pm 1.3 . A rapid rise and recovery to eventual values of $\mathrm{PCr}$ and ATP, as well as normalization of $\mathrm{pH}$, occur by $7.5 \mathrm{~min}$ of reperfusion, to $93 \pm 7 \%$ and $41 \pm 4 \%$ of baseline levels and to $7.22 \pm .03$, respectively. During the next $35 \mathrm{~min}$ of reperfusion, when percent developed pressure falls and slowly recovers, global levels of high-energy phosphates and intracellular $\mathrm{pH}$ do not change. Therefore, as is the case in the initial series of hearts at the faster heart rate, delayed mechanical recovery after ischemia cannot be explained by changes in global levels of high-energy phosphates or $\mathrm{pH}$ correction since these attain their eventual levels within the early minutes of reperfusion. The major point to note in the figure is that the peak SLIF is temporally correlated with both the fall in developed pressure and the peak increase in EDP during early reperfusion when the metabolic variables are stable and that SLIF and EDP subsequently decrease while developed pressure increases during later reperfusion during continued metabolic stability.

The time course of contractile and SLIF recovery during 

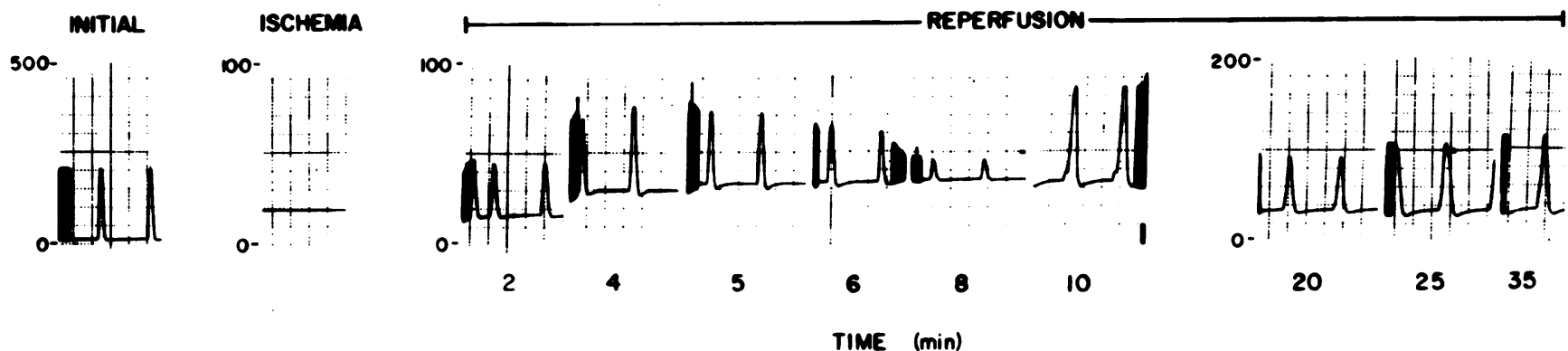

TIME (min)

Figure 5. Representative example of contractile function before, during, and after $65 \mathrm{~min}$ of ischemia at a heart rate of 20 beats $/ \mathrm{min}$. Developed pressure declines during minutes 5-8 of reperfusion and then slowly increases over the next $30 \mathrm{~min}$.

reperfusion suggests that altered calcium loading may account for the delayed contractile recovery. Further experiments were therefore conducted to evaluate the effect of cell calcium loading on SLIF and systolic and diastolic function. Studies were performed to first assess the effect of calcium overload on developed pressure, EDP, and SLIF under these specific experimental conditions during normal perfusion and then to assess the effect of altered calcium concentration on reperfusion SLIF, mechanical recovery, and cellular calcium.

Under conditions of normal perfusion, simultaneous SLIF, developed pressure, and EDP were measured over a range of perfusate calcium concentrations in three beating hearts. The average results are presented in Fig. $7 \mathrm{~A}$. Developed pressure rises rapidly over the perfusate calcium range of $0.25-4 \mathrm{mM}$. Continued increases in perfusate calcium to $15 \mathrm{mM}$, however, cause a decrease in developed pressure to $75 \%$ of its maximum. Both EDP and SLIF increase significantly to $17 \pm 3 \mathrm{~mm} \mathrm{Hg}$ and $60 \pm 7 \mathrm{~Hz}$, respectively $(P<0.05$, both), with increases in perfusate calcium from 0.25 to $15 \mathrm{mM}$. Fig. $7 \mathrm{~B}$ shows a pressure tracing and SLIF (hertz) for one heart in which the perfusate calcium was decreased from 15 to $0.5 \mathrm{mM}$ at the end of the experiment. The transient rise and subsequent fall in developed pressure during steadily decreasing SLIF induced by lowered calcium demonstrate that the relationship between these parameters and calcium concentration remains bidirectionally intact and that calcium overload is at least partially reversible in this setting. Thus, with normal perfusion, calcium overload elevates EDP and depresses developed pressure and is associated with excessive calcium oscillations as indexed by SLIF.

Since depressed developed pressure, elevated EDP, and excessive SLIF occur during calcium overload in the setting of normal perfusion and also in the setting of postischemic reperfusion, further experiments were performed during which cellular calcium was altered by changing perfusate calcium during reperfusion in order to assess the subsequent effects on mechanical function, SLIF, and cellular calcium content. Preliminary studies demonstrated that reperfusion with a perfusate calcium concentration of $0 \mathrm{mM}$ abolished SLIF. Since the absence of perfusate calcium abolishes mechanical systoles and subsequent reintroduction of calcium results in the calcium paradox, this model did not permit the study of mechanical function. Therefore two additional groups, consisting of four hearts each, differing only in perfusate calcium concentration ( 1.5 or $0.08 \mathrm{mM}$ ) during the first $10 \mathrm{~min}$ of reperfusion, were studied. The ischemic duration and conditions were the same as those in the earlier experiments with the exception that the ventricular balloon was deflated during the first $10 \mathrm{~min}$ of reperfusion in both groups to control for differ- ences in cardiac work which would otherwise occur during this period. The perfusate also contained $1 \mathrm{mM} \mathrm{KCoEDTA}$ as an extracellular marker and $2 \mathrm{mM}$ phosphate.

Before ischemia and during normal calcium perfusion, initial SLIF (18 \pm 2 and $23 \pm 3 \mathrm{~Hz}$ ) and developed pressure $(181 \pm 7$ and $161 \pm 22 \mathrm{~mm} \mathrm{Hg}$ ) did not differ significantly between the eventual normal and low reperfusion calcium groups. Fig. 8 presents SLIF as percentage of baseline for both groups vs. reperfusion time in minutes. As in the other experiments conducted with $1.5 \mathrm{mM}$ perfusate calcium, SLIF peak at 7-8 min of reperfusion at nearly three times baseline values and subsequently decline steadily. However, the hearts reperfused initially with $0.08 \mathrm{mM}$ calcium do not demonstrate a SLIF "overshoot" but rather have slowly increasing SLIF which only approach baseline values at the time of reintroduction of $1.5 \mathrm{mM}$ calcium at $10 \mathrm{~min}$. Thereafter, SLIF plateaued in this group. Fig. 9 shows the percent developed pressure and cell calcium content measured at the end of reperfusion. Percent developed pressure was significantly higher $(74 \pm 3 \%$ vs. $39 \pm 13 \%, P<0.05)$ in hearts transiently reperfused with low calcium whereas EDP trended lower $(35 \pm 7$ vs. $53 \pm 8 \mathrm{~mm} \mathrm{Hg}$, $P=0.1$ ). Cellular calcium, measured at the end of the $20-\mathrm{min}$ reperfusion period, is significantly lower $(5.9 \pm .3$ vs. $10.3 \pm 1.4$ $\mu \mathrm{mol} / \mathrm{g}$ dry wt, $P<0.05$ ) in hearts initially reperfused with low perfusate calcium. Thus, prevention of reperfusion-induced cellular calcium overload with reduced perfusate calcium results in concomitant abolishment of excess calcium oscillations, as indexed by SLIF, and improved mechanical activity.

\section{Discussion}

In the present study one group of isolated rat hearts stimulated at 200 beats/min underwent $40 \mathrm{~min}$ of global, no-flow ischemia at $30^{\circ} \mathrm{C}$. This resulted in a rapid cessation of systolic pressure development, a subsequent rise in EDP from a mean of 12 to $28 \mathrm{~mm} \mathrm{Hg}$, a fall in $\mathrm{PCr}$ so as to be nearly undetectable, a fall in ATP to $20 \%$ of its baseline value, and a decline in cellular $\mathrm{pH}$ to 6.1. This profile was mimicked in another group of hearts stimulated at a rate of 20 beats/min, accomplished with atrioventricular block, at the end of $65 \mathrm{~min}$ of ischemia. SLIF measured in the latter group also fell to undetectable levels during the early ischemic period. During reperfusion in both groups there was a monotonic increase in ATP, PCr, and $\mathrm{pH}$, which fully recovered to their steady values within 2-5 min of restoration of normal flow. While metabolic recovery was associated with a rapid initial recovery of contractile function, an abrupt decline accompanied by an increase in EDP occurred after about 5 min after reflow. This was not accom- 


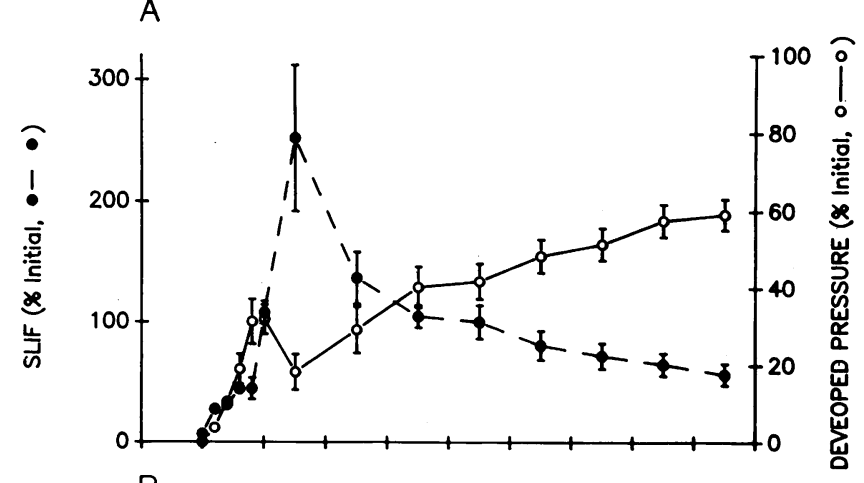

B
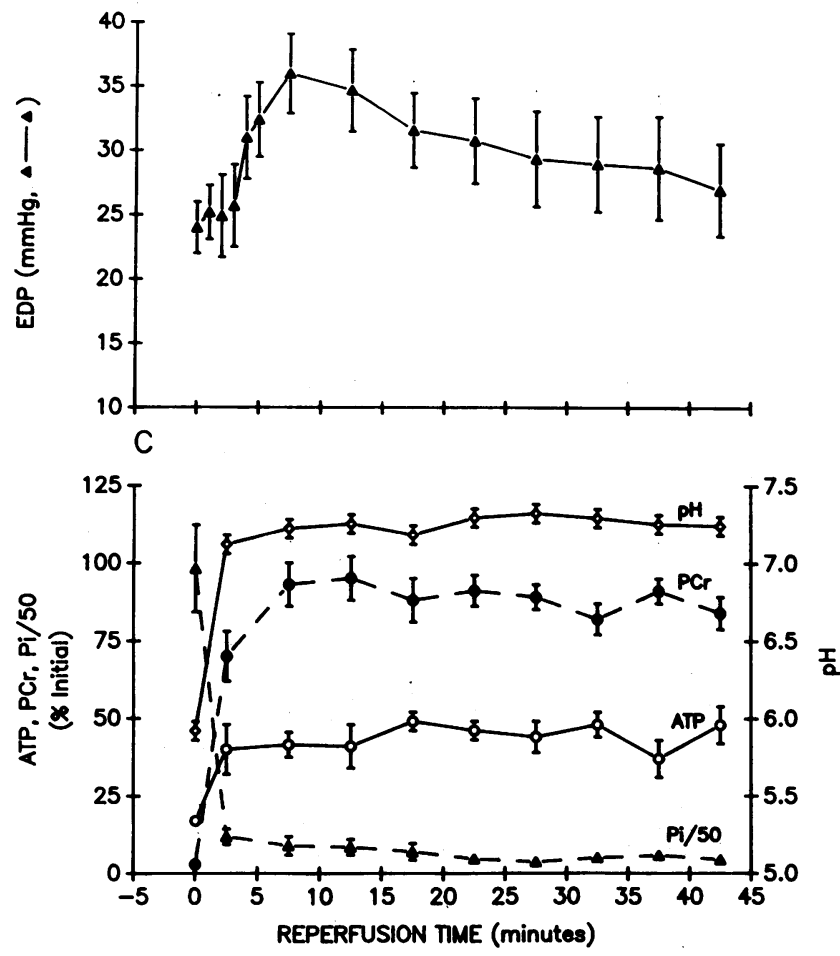

Figure 6. Contractile and metabolic recovery and diastolic $\mathrm{Ca}^{++}$ oscillations (SLIF) during reperfusion (heart rate $=20$ beats $/ \mathrm{min}$ ). Mean values of developed pressure (as percent initial, $n=10$ ), EDP (mm Hg, $n=10)$, and $\operatorname{SLIF~}(\mathrm{Hz}, n=5)$ are plotted in $A$ vs. reperfusion time ( $\mathrm{min}$ ). Developed pressure recovers during the first $5 \mathrm{~min}$ of reperfusion but then abruptly falls, reaching a nadir at $7.5 \mathrm{~min}$. Thereafter, developed pressure increases slowly, attaining $60 \%$ of its initial value after $45 \mathrm{~min}$ of reperfusion. $(B)$ EDP climbs rapidly in the first minute of reperfusion possibly, because of the "garden hose" effect. EDP reaches a maximum at $7.5 \mathrm{~min}$ of reperfusion, which is significantly higher than that at 2 min of reperfusion $(P<0.01)$ and which occurs as developed pressure is falling. The EDP data during reperfusion are well fit by a third degree polynomial $(P<0.008)$ with an intercept of 23.4 and coefficients of $1.90,-0.92$, and 0.001 . PCr, ATP, and $P_{i}$ are presented as percentage of initial, preischemic values. The mean preischemic values of $P C r / A T P$ and $P_{i} / A T P$ were $2.02 \pm 0.10$ and $0.10 \pm 0.01$, respectively. $(C)$ Metabolic parameters $(n$ $=5$ ) recover to their full extent within 5-7.5 min of reperfusion and then plateau, similar to that seen at the faster stimulation rate (Fig. 3 ), and at a time when contractile function is falling. Diastolic $\mathrm{Ca}^{++}$ oscillations, indexed by SLIF, increase from the end-ischemic value of zero and peak at a mean value of three times baseline at $7.5 \mathrm{~min}$ of reperfusion. This SLIF peak coincides with the transient fall in developed pressure and the peak in EDP. Subsequently, SLIF decrease monotonically over the next $35 \mathrm{~min}$ of reperfusion while developed pressure recovers.
A

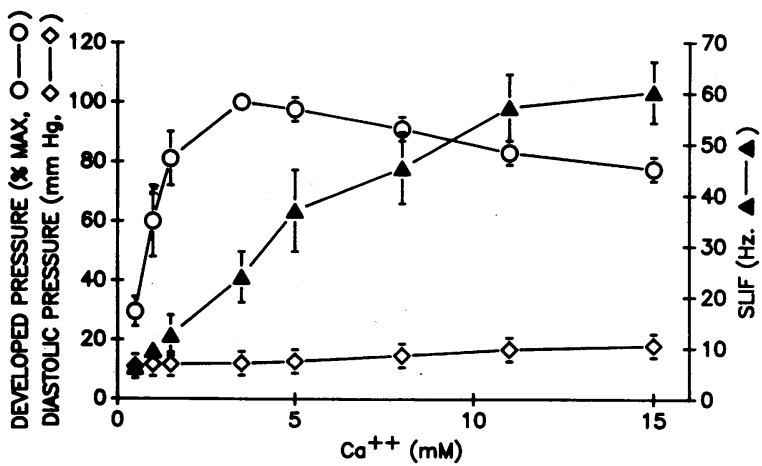

B

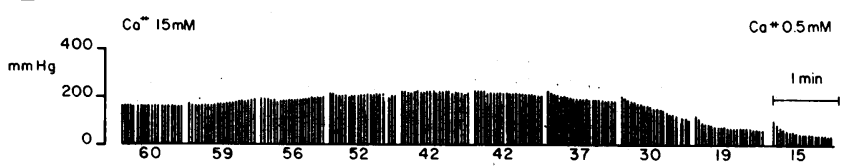

Figure 7. Effects of altered perfusate $\mathrm{Ca}^{++}$on SLIF, developed pressure, and EDP in the absence of ischemia/reperfusion. $(A)$ Developed pressure increases with increases in perfusate $\mathrm{Ca}^{++}$. between 0.25 and $4 \mathrm{mM}$. At progressively higher levels of perfusate $\mathrm{Ca}^{++}$to $15 \mathrm{mM}$, developed pressure falls. SLIF and EDP increase as perfusate $\mathrm{Ca}^{++}$is increased. Using two-way. ANOVA for the entire data set there was a significant interaction of EDP with perfusate $\left[\mathrm{Ca}^{++}\right], P<0.05$. Linear regression of the EDP data produced an intercept value of 11 and coefficient (slope) of $0.389(P<0.05)$. Note that the highest SLIF are seen at high cellular $\mathrm{Ca}^{++}$levels and are associated with depressed developed pressure. (B) Contractile function and concurrent SLIF measures presented under the developed pressure tracing in a heart in which perfusate $\mathrm{Ca}^{++}$was lowered from 15 to $0.3 \mathrm{mM}$. It demonstrates reversibility of the high $\mathrm{Ca}^{++}$-induced depression in contractile function and that the direct relationship between cell $\mathrm{Ca}^{++}$and SLIF is bidirectionally intact.

panied by a decline in metabolic status, which remained stable (Figs. $3 B$ and $6 C$ ). This decline in contractile function was followed by a more gradual recovery, which did not achieve its final value until $\sim 30 \mathrm{~min}$ after the onset of reperfusion. The recovery of contractile function was associated with a decrease in EDP. SLIF also recovered during reperfusion with the time course of the initial recovery paralleling the recovery of contractile function (Fig. $6 A$ ). However, at a time when developed pressure began its decline, SLIF continued to increase and exceeded its baseline value by a mean of $250 \%$. After this overshoot, a decline in SLIF occurred over $30 \mathrm{~min}$, paralleling the gradual secondary recovery in developed pressure.

Contractile failure after early contractile recovery. Although the abrupt decline in developed pressure that followed the initial early recovery cannot be attributed to a reversal in the recovery of metabolic parameters, it may be linked to the marked overshoot of SLIF. SLIF are due to cytosolic calcium oscillations and depend not only upon cytosolic calcium loading (18) but also upon sarcoplasmic reticulum function and myofilament calcium responsiveness. The myofilament response to calcium may be altered by changes in $\mathrm{pH}$, in $\mathrm{P}_{\mathrm{i}}(4-9)$, and in myofilament integrity itself. The reversible decline in SLIF present at the end of the ischemic portion of the protocol may be attributed to decreased sarcoplasmic reticulum function related to the decline in high-energy phosphates and/or to 


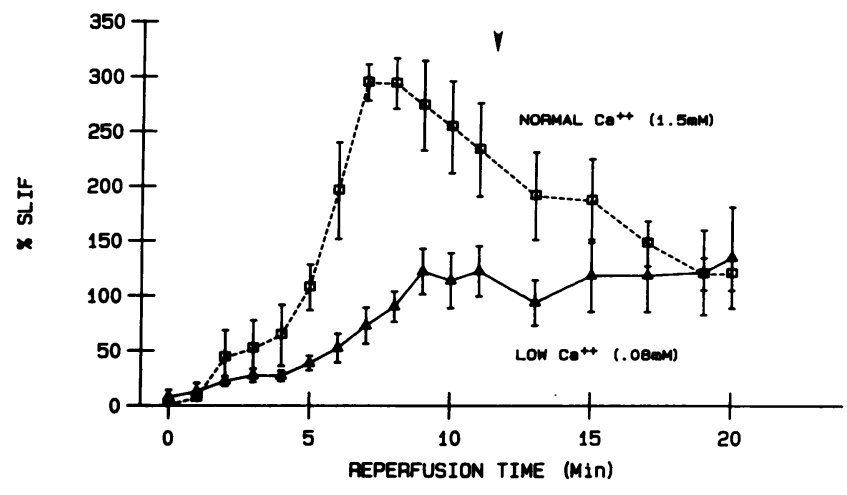

Figure 8. SLIF during reperfusion with low $(0.08 \mathrm{mM})$ and normal $(1.5 \mathrm{mM}) \mathrm{Ca}^{++}$-containing solutions. In hearts reperfused with low $\mathrm{Ca}^{++}$perfusate $(n=4)$ after 65 min of no-flow ischemia, SLIF increase slowly and do not "overshoot" to three times the baseline values as seen in hearts reperfused with normal $\mathrm{Ca}^{++}$-containing solution $(n=4)$. SLIF levels are similar at $15 \mathrm{~min}$ of reperfusion in both groups when perfusate $\mathrm{Ca}^{++}$is $1.5 \mathrm{mM}$ in all hearts. The arrow indicates when perfusate calcium of $1.5 \mathrm{mM}$ was restored in the low calcium $(0.08 \mathrm{mM})$ group.

decreased calcium-myofilament interaction owing to the fall in $\mathrm{pH}$ and/or rise in inorganic phosphate. It is probably not due to a decline in cytosolic calcium inasmuch as recent studies indicate that free cytosolic calcium rises during ischemic conditions and during metabolic inhibition (33-36). There is a
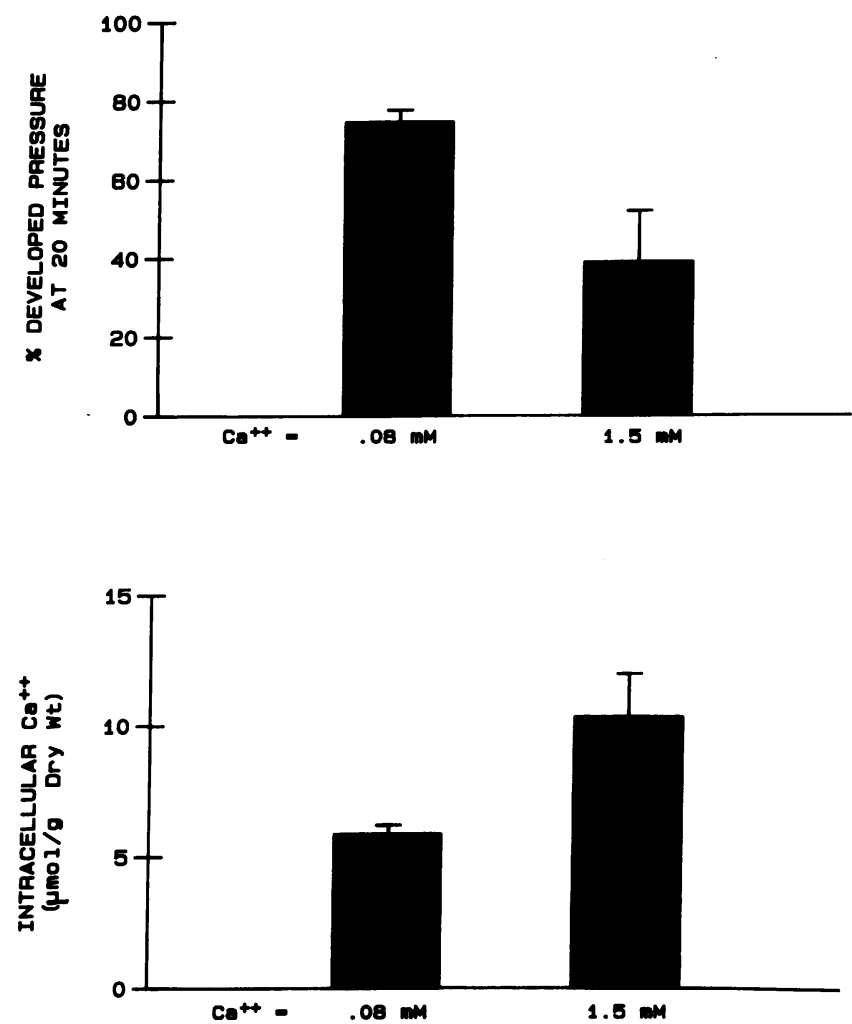

Figure 9. Developed pressure and cellular calcium following reperfusion with normal and low calcium-containing perfusate. Hearts reperfused initially with $1.5 \mathrm{mM} \mathrm{Ca}^{++}$had significantly higher cell calcium and lower developed pressure at 20 min than hearts perfused initially (0-10 min of reperfusion) with low $(0.08 \mathrm{mM}) \mathrm{Ca}^{++}$-containing solution. marked rise and reversible SLIF overshoot after 5-7 min of early reperfusion. SLIF recovery during reperfusion may reflect recovery of sarcoplasmic reticulum function or myofilament responsiveness as well as cell calcium loading at that time. To the extent that the first two factors are related to metabolic recovery, it should be noted that although inorganic phosphate falls and $\mathrm{pH}$ and high-energy phosphate rise during early reperfusion. Intracellular $\mathrm{pH}, \mathrm{ATP}$, and $\mathrm{PCr}$ have all recovered to their fullest extent and are not changing while SLIF is exhibiting the overshoot and then declining. Although ${ }^{31} \mathrm{P}$ NMR methods index global levels of these compounds, which may not reflect local sarcoplasmic reticulum or myofilament levels, it is more likely that the SLIF overshoot is due to high cytosolic calcium. It has been shown that a marked total cell calcium gain occurs during early reoxygenation of hypoxic tissue (37) as well as during reperfusion of previously ischemic myocardium $(10,11)$. A marked increase in spontaneous oscillatory contractile waves, reflecting spontaneous sarcoplasmic reticulum calcium release, has been directly observed in individual rat ventricular cells during reoxygenation (38). Thus, the overshoot of SLIF during reperfusion most likely results from excessive myofilament-sarcoplasmic reticulum calcium cycling due to cell calcium overload. Diastolic calcium oscillations have also been detected as oscillations in aequorin luminescence after the restoration of oxidative metabolism by the removal of cyanide in isolated, glycogen-depleted, ferret ventricular muscle (36). In that study it was shown that the calcium oscillations occurred at the time when average cytosolic calcium exhibited its peak overshoot. The maximum increase in SLIF in our experiments occurred 5-7 min after reperfusion, which is slower than that seen for maximum calcium accumulation in the study referred to above (36). This difference in the apparent time course of calcium accumulation may be related to differences in species, in the severity of the metabolic insult, or to the slower pacing rate used in our experiments. Thus cytosolic calcium overload during reperfusion appears to be the most likely mechanism responsible for exacerbating or causing de novo diastolic calcium oscillations which results in SLIF overshoot.

Prior studies have suggested that calcium overload after ischemia or hypoxia can be modulated by interventions that reduce sarcolemmal calcium movement, though not necessarily that which occurs via electrogenic calcium channels (39-41). The present results indicate that calcium overload during reperfusion can be prevented by reperfusing with low calcium-containing perfusate. This maneuver also prevents the overshoot of SLIF. These data further support the notion that excessive SLIF during reflow, at a time when $\mathrm{pH}$, inorganic phosphate, and high-energy phosphates are constant, reflects the occurrence of cell calcium overload. The results of additional protocols in the same experimental model in the present study and in previous studies (19-21) indicate that calcium overload and an increase in SLIF oscillations in the absence of ischemia and reperfusion can also be associated with a decline in systolic function.

The present results indicate that excessive sarcoplasmic reticulum calcium oscillations, reported as an overshoot in SLIF, occur at a time when the rate of contractile recovery slows and reverses. There are several mechanisms by which oscillatory spontaneous diastolic sarcoplasmic reticulum release may alter stimulated developed pressure. Spontaneous calcium release results in inhomogeneous diastolic sarcoplasmic reticulum calcium loading and heterogeneous diastolic 
sarcomere lengths, leading to inhomogeneous calcium release and inhomogeneous myofilament activation $(16,19-21)$ in the subsequent systole. In addition, spontaneous diastolic sarcoplasmic reticulum calcium release could result in less action potential-generated calcium flux in the subsequent systole because of calcium-dependent inactivation of L-type calcium currents. Finally the areas throughout the tissue where there is reduced calcium release will act as compliant "islands" diminishing the tension the total muscle will develop (16).

Late contractile recovery. After the rapid SLIF increase which coincided with an interruption in developed pressure recovery, there was a decline in SLIF toward its baseline level, and at the end of the 30-min recovery period SLIF had fallen to below its baseline value. This cannot be attributed to an increase in $\mathrm{pH}$ or a decline in $\mathrm{P}_{\mathrm{i}}$ in that the NMR parameters indicate that these are not changing at this time. It is more likely that this can be related to the calcium load being pumped out of some cells and/or ATP depletion and death of cells with extreme calcium overload. An increase in developed pressure parallels the SLIF decline. At 30-min after reflow developed pressure was $\sim 40 \%$ of its initial value and was associated with a cellular calcium content of $10.3 \mu \mathrm{mol} / \mathrm{g}$ dry weight. Compared with early reperfusion with normal perfusate calcium of $1.5 \mathrm{mM}$, perfusion with low calcium during the first 10 min of reperfusion resulted in a halving of the calcium content at the end of the reperfusion period, retarded the recovery of SLIF during early reperfusion, and prevented the SLIF overload, all of which suggest that the calcium overload occurred during early reperfusion. The initial calcium overload was probably more marked than that measured at $30 \mathrm{~min}$ in the present study because recent studies suggest that eventual functional recovery is paralleled by a reduction in the average cytosolic calcium (36). In the present study, developed pressure at the end of the reperfusion period was improved in the group receiving low calcium during early reperfusion, recovering to a mean of $74 \%$ of its initial value. While initial perfusion with low calcium suppressed the marked early SLIF overshoot, it did not prevent the slow recovery of SLIF back to its preischemic level $30 \mathrm{~min}$ after reflow and SLIF did not differ between the calcium groups $30 \mathrm{~min}$ after reperfusion. Thus, while excessive overshoot of calcium oscillations during early reperfusion can limit functional recovery, i.e., contribute to myocardial "stunning," the level of calcium oscillations present at the end of the reperfusion period in the present study is not a determinant of late functional recovery although the tissue calcium load is related to late functional recovery. This suggests that, in addition to causing sarcoplasmic reticulum myofilament oscillations during early reflow, calcium overload in some cells is sufficient to cause permanent injury.

\section{Acknowledgments}

We wish to thank Mrs. Spring Metcalf for assistance in the preparation of the manuscript.

This work was supported in part by grant HL-17655-14 from the National, Heart, and Blood Institute Specialized Center of Research.

\section{References}

1. Matsuzaki, M., K. P. Gallagher, W. S. Kemper, F. White, and J. Ross, Jr. 1983. Sustained regional dysfunction produced by prolonged coronary stenosis: gradual recovery after reperfusion. Circulation. 68:170-182.

2. Braunwald, E., and R. A. Kloner. 1982. The stunned myocar- dium: prolonged, postischemic ventricular dysfunction. Circulation. 66:1146-1149.

3. Flaherty, J. T., M. L. Weisfeldt, B. H. Bulkley, T. J. Gardner, V. L. Gott, and W. E. Jacobus. 1982. Mechanism of myocardial cell damaged assessed by phosphorus-31 nuclear magnetic resonance. Circulation. 65:561-571.

4. Donaldson, S. K. B., and L. Hermansen. 1978. Differential, direct effects of $\mathrm{H}^{+}$on $\mathrm{Ca}^{2+}$-activated force of skinned fibers from the soleus, cardiac, and adductor magnus muscles of rabbits. Pflügers Arch. Eur. J. Physiol. 376:55-65.

5. Blanchard, E. M., and R. J. Solaro. 1984. Inhibition of the activation and troponin calcium binding of dog cardiac myofibrils by acidic pH. Circ. Res. 55:382-391.

6. Fabiato, A., and F. Fabiato. 1978. Effects of $\mathrm{pH}$ on the myofilaments and the sarcoplasmic reticulum of skinned cells from cardiac and skeletal muscle. J. Physiol. (Lond.). 276:233-255.

7. Kentish, J. C. 1986. The effects of inorganic phosphate and creatine phosphate on force production in skinned muscles from rat ventricle. J. Physiol. (Lond.). 370:585-604.

8. Hibberd, M. G., J. A. Dantzig, D. R. Trentham, and Y. E. Goldman. 1985. Phosphate release and force generation in skeletal muscle fibers. Science (Wash. DC). 288:1317-1319.

9. Herzig, J. W., J. W. Peterson, J. C. Ruegg, and R. J. Solaro. 1981. Vanadate and phosphate ions reduce tension and increase cross-bridge kinetics in chemically skinned heart muscle. Biochim. Biophys. Acta. 672:191-196.

10. Henry, P. D., R. Schuchleib, J. Davis, E. S. Weiss, and B. E. Sobel. 1977. Myocardial contracture and accumulation of mitochondrial calcium in ischemic rabbit heart. Am. J. Physiol. 233:H677H684.

11. Shine, K. I., A. M. Douglas, and N. V. Ricchiuti. 1978. Calcium, strontium, and barium movements during ischemia and reperfusion in rabbit ventricle. Circ. Res. 43:712-720.

12. Kusuoka, H., J. K. Porterfield, H. F. Weisman, M. L. Weisfeldt, and E. Marban. 1987. Pathophysiology and pathogenesis of stunned myocardium. J. Clin. Invest. 79:950-961.

13. Jacobus, W. E., G. J. Taylor, D. P. Hollis, and R. L. Nunnally, 1977. Phosphorus nuclear magnetic resonance of perfused working rat hearts. Nature (Lond.). 265:756-758.

14. Stern, M. D., A. A. Kort, C. M. Bhatnager, and E. G. Lakatta. 1983. Scattered-light intensity fluctuations in diastolic rat cardiac muscle caused by spontaneous $\mathrm{Ca}^{+2}$-dependent cellular mechanical oscillations. J. Gen. Physiol. 82:119-153.

15. Stern, M. D., H. F. Weisman, D. G. Renlund, G. Gerstenblith, and E. G. Lakatta. 1985. Cellular calcium oscillations in intact perfused hearts detected by laser light scattering: cellular mechanism for diastolic tone. Circulation. 72(Suppl. III):296. (Abstr.)

16. Kort, A. A., and E. G. Lakatta. 1984. Calcium dependent mechanical oscillations occur spontaneously in unstimulated mammalian cardiac muscle. Circ. Res. 54:396-404.

17. Capogrossi, M. C., A. A. Kort, H. A. Spurgeon, and E. G. Lakatta. 1986. Single adult rabbit and rat cardiac myocytes retain the $\mathrm{Ca}^{2+}$ - and species-dependent systolic and diastolic contractile properties of intact muscle. J. Gen. Physiol. 88:589-613.

18. Kort, A. A., M. C. Capogrossi, and E. G. Lakatta. 1985. Frequency, amplitude, and propagation velocity of spontaneous $\mathrm{Ca}^{2+}$-de. pendent contractile waves in intact adult rat cardiac muscle and isolated myocytes. Circ. Res. 57:844-855.

19. Lakatta, E. G., and D. L. Lappe. 1981. Diastolic scattered light fluctuation, resting force and twitch force in mammalian cardiac muscle. J. Physiol. (Lond.). 315:369-394.

20. Lakatta, E. G., M. C. Capogrossi, H. A. Spurgeon, and M. D. Stern. 1989. Characteristics and functional implications of spontaneous sarcoplasmic reticulum generated cytosolic calcium oscillations in myocardial tissue. In Cell Calcium Metabolism. G. Fiskum G, editor. Plenum Press, New York. 529-543.

21. Kort, A. A., and E. G. Lakatta. 1988. Spontaneous sarcoplasmic reticulum calcium release in rat and rabbit cardiac muscle: rela- 
tion to transient and rested-state twitch tension. Circ. Res. 63:969979.

22. Allen, D. G., D. A. Eisner, J. S. Pirolo, and G. L. Smith. 1985 The relationship between intracellular calcium and contraction in calcium overloaded ferret papillary muscles. J. Physiol. (Lond.). 364:169-182.

23. Gadian, D. G., G. K. Radda, R. E. Richards, and P. J. Seeley. 1979. 31-P NMR in living tissue: the road from a promising to an important tool in biology. In Biological Applications of Magnetic Resonance. R. G. Shulman, editor. Academic Press, Inc., New York. 463-535.

24. Morris, G. M., D. G. Allen, and C. L. Orchard. 1984. High time resolution of 31-P NMR studies of the perfused rabbit heart. $A d v$. Myocardiol. 6:27-38.

25. Hoerter, J. A., M. V. Miceli, W. E. Jacobus, D. G. Renlund, G. Gerstenblith, and E. G. Lakatta. 1986. A 31-P study of the metabolic, contractile and ionic consequences of induced $\mathrm{Ca}^{2+}$ alterations in the isovolumic rat heart. Circ. Res. 58:539-551.

26. Jacobus, W. E., I. H. Pores, S. K. Lucas, C. H. Kallman, M. L. Weisfeldt, and J. T. Flaherty. 1982. The role of intracellular pH in the control of normal and ischemic myocardial contractility: $\mathrm{a}^{31} \mathrm{P}$ nuclear magnetic resonance and mass spectroscopic study. In Intracellular pH: Its Measurement, Regulation, and Utilization in Cardiac Function. R. Nuccitelli and D. W. Deaner, editors. Alan R. Liss, Inc., New York. 537-565.

27. Saleh, B. 1978. Photoelectric statistics with application to spectroscopy and optical communications. In Springer Series in Optical Science. Volume 6. Springer-Verlag, Berlin. 190-338.

28. Bridge, J. H. B., M. M. Bersohn, F. Gonzalez, and J. B. Bassingthwaighte. 1982. Synthesis and use of radiocobaltic EDTA as an extracellular marker in rabbit heart. Am. J. Physiol. 242:H671-H676.

29. Ruano-Arroyo, G., G. Gerstenblith, and E. G. Lakatta. 1984. "Calcium-paradox" in the heart is modulated by Na during the calcium free period. J. Mol. Cell. Cardiol. 16:783-793.

30. Renlund, D. G., E. G. Lakatta, E. D. Mellits, and G. Gerstenblith. 1985. Calcium-dependent enhancement of myocardial diastolic tone and energy utilization dissociates systolic work and oxygen consumption during low sodium perfusion. Circ. Res. 57:876-888.
31. Dwyer, F. P., E. Gyarfas, and D. Mellor. 1965. The resolution of racemization of potassium ethylenediamine tetraacetate cobaltate. III. J. Physiol. Chem. 59:296-297.

32. Renlund, D. G., G. Gerstenblith, H. F. Weisman, E. G. Lakatta, and M. D. Stern. 1985. Mechanical manifestations of sarcoplasmic-reticulum $\mathrm{Ca}^{++}$cycling are markedly perturbed by ischemia. Circulation. 72(Suppl. III):356. (Abstr.)

33. Steenbergen, C., E. Murphy, L. Levy, and R. E. London. 1987. Elevation of cytosolic free calcium concentration early in myocardial ischemia in perfused rat heart. Circ. Res. 60:700-707.

34. Lee, H. C., R. Mohabir, N. Smith, M. R. Franz, and W. T. Clusin. 1988. Effect of ischemia on calcium-dependent fluorescence transients in rabbit hearts containing Indo 1. Circulation. 78:10471059.

35. Marban, E., M. Kitakaze, H. Kusuoka, J. P. Porterfield, D. T. Yue, and V. P. Chacko. 1987. Intracellular free calcium concentration measured with ${ }^{19} \mathrm{~F}$ NMR spectroscopy in intact ferret hearts. Proc. Natl. Acad. Sci. USA. 84:6005-6009.

36. Smith, G. L., and D. G. Allen. 1988. The effects of metabolic blockade on intracellular calcium concentration in isolated ferret ventricular muscle. Circ. Res. 62:1223-1236.

37. Lakatta, E. G., Nayler, W. G., and P. A. Poole-Wilson. 1979. Calcium overload and mechanical function in posthypoxic myocardium: biphasic effect of pH during hypoxia. Eur. J. Cardiol. 10:77-87.

38. Stern, M. D., A. M. Chien, M. C. Capogrossi, D. J. Pelto, and E. G. Lakatta. 1985. Direct observation of the "oxygen paradox" in single rat ventricular myocytes. Circ. Res. 56:899-903.

39. Allen, D. G., and C. H. Orchard. 1987. Myocardial contractile function during ischemia and hypoxia. Circ. Res. 60:153-168.

40. Koomen, J. M., J. A. M. Schevers, and J. Noordhoek. 1983. Myocardial recovery from global ischemia and reperfusion: effects of pre- and/or post-ischemic perfusion with low-Ca ${ }^{2+}$. J. Mol. Cell. Cardiol. 15:383-391.

41. Kuroda, H., S. Ishiguro, and T. Mori. 1986. Optimal calcium concentration in the initial reperfusate for post-ischemic myocardium performance (calcium concentration during reperfusion). J. Mol. Cell. Cardiol. 18:625-633. 